\title{
Using the MEM-net program to report on mapping the EchoColorDoppler assessment for chronic cerebro spinal venous insufficiency
}

This article was published in the following Dove Press journal:

Journal of Vascular Diagnostics

10 July 2014

Number of times this article has been viewed

\author{
Sandro Mandolesi' \\ Aldo d'Alessandro \\ Ettore Manconi ${ }^{3}$ \\ Tarcisio Niglio ${ }^{4}$ \\ Augusto Orsini ${ }^{5}$ \\ Dimitri Mandolesi ${ }^{6}$ \\ Alessandro d'Alessandro ${ }^{7}$ \\ Francesco Fedele \\ 'Department of Cardiovascular \\ and Respiratory Sciences, Sapienza \\ University Rome, Rome, Italy; \\ ${ }^{2}$ Department of Angiology, \\ "T Masselli-Mascia" Hospital, \\ San Severo (FG), Foggia, Italy; \\ ${ }^{3}$ Department of Cardiovascular \\ and Neurological Sciences, University \\ of Cagliari, Cagliari, Italy; ${ }^{4}$ Istituto \\ Superiore di Sanità, Rome, Italy; \\ ${ }^{5}$ Department of Vascular Surgery, \\ "Gioia" Hospital, Sora (FR), Sora, \\ Italy; 'Medicina del lavoro Sapienza \\ University Roma, Rome, Italy; \\ ${ }^{7}$ Faculty of Medicine, Catholic \\ University "Our Lady of Good \\ Counsel," Tirana, Albania
}

Correspondence: Sandro Mandolesi Via San Martino della Battaglia 25,

Rome 00185, Italy

Tel +3933565I2303

Fax +39064873984

Email s.mandolesi@email.it
Introduction: Chronic cerebrospinal venous insufficiency (CCSVI) is characterized by multiple stenosis/obstructions affecting the principal extracranial outflow pathways of the cerebrospinal venous system. Using EchoColorDoppler (ECD) to assess chronic CCSVI is a very difficult and long examination. It takes about an hour even for an expert sonographer.

Methods: Hemodynamic morphological map (MEM-Net) is a program that works on the Internet. All the morphological and hemodynamic data of the patient can be entered into the program's anatomical scheme to create a map of the ECD report. The program also allows us to collect all the data during the ECD assessment and, using its algorithm, make the report uniform.

Conclusion: Reporting on the map by using MEM-net shortens the time of ECD written reporting that is done automatically. The program also makes a blind control of the report and enables the use of it for scientific research. We hope that in the future everyone will use this data collection tool for all scientific work on this topic.

Keywords: CCSVI, ECD, Map, cerebral venous system, sonography

\section{Introduction}

Chronic cerebrospinal venous insufficiency (CCSVI) is characterized by multiple stenosis/obstructions affecting the principal extracranial outflow pathways of the cerebrospinal venous system, the internal jugular veins (IJVs), and the azygos vein, distributed in four main hemodynamic patterns. ${ }^{1}$

Furthermore, CCSVI determines significant changes in the cerebral venous hemodynamic, with a very high incidence of reflux in both intracranial and extracranial venous segments and loss of the postural regulation of cerebral venous outflow. ${ }^{1,2}$ Recently, Zamboni et al ${ }^{1}$ suggested five EchoColorDoppler (ECD) venous criteria that characterize this syndrome: 1) reflux constantly present in an outflow pathway of IJVs or vertebral veins (VVs) assessed in supine and standing posture $\left(0^{\circ}\right.$ and $\left.90^{\circ}\right) ; 2$ ) reflux propagated upward to the deep cerebral veins (DCVs), including superficial or internal cerebral vein, basal vein, Galen vein; 3) evidence of IJVs stenosis; 4) flow not Doppler detectable in the IJVs and VVs assessed in supine and standing posture $\left(0^{\circ}\right.$ and $90^{\circ}$ ) and ; 5) negative difference in cross sectional area (CSA) of the IJV assessed in supine and standing posture $\left(0^{\circ}\right.$ and $\left.90^{\circ}\right)$ in the IJV $(\Delta \mathrm{CSA}) .^{3}$ The presence of two of them is enough to diagnose CCSVI.

Using ECD to assess CCSVI is a very difficult and long examination, requiring about an hour of work even for an expert sonographer. It involves a venous ECD assessment of the main veins draining the brain in the supine position, a venous ECD assessment in the upright position, a Manconi breathing test, a neck rotation test, 
VENOUS ECHOCOLORDOPPLER

DIPARTIMENTO DI SCIENZE

CARDIOAGSCOLARI RESPIRATOK

EMORFOLOGICHE

(10) SAPIENZA

CCSVI "SAPIENZA" CENTER

Viale del Policlinico, 155 -00161 Tel. 0649979001

e-mail: s.mandolesi@email.it

Supine : red color

Upright : green color

\begin{tabular}{|c|c|}
\hline \multicolumn{1}{|c|}{ CSA } \\
\hline Dx & Sx \\
\hline 120,00 & 150,00 \\
\hline 45,00 & 56,00 \\
\hline
\end{tabular}

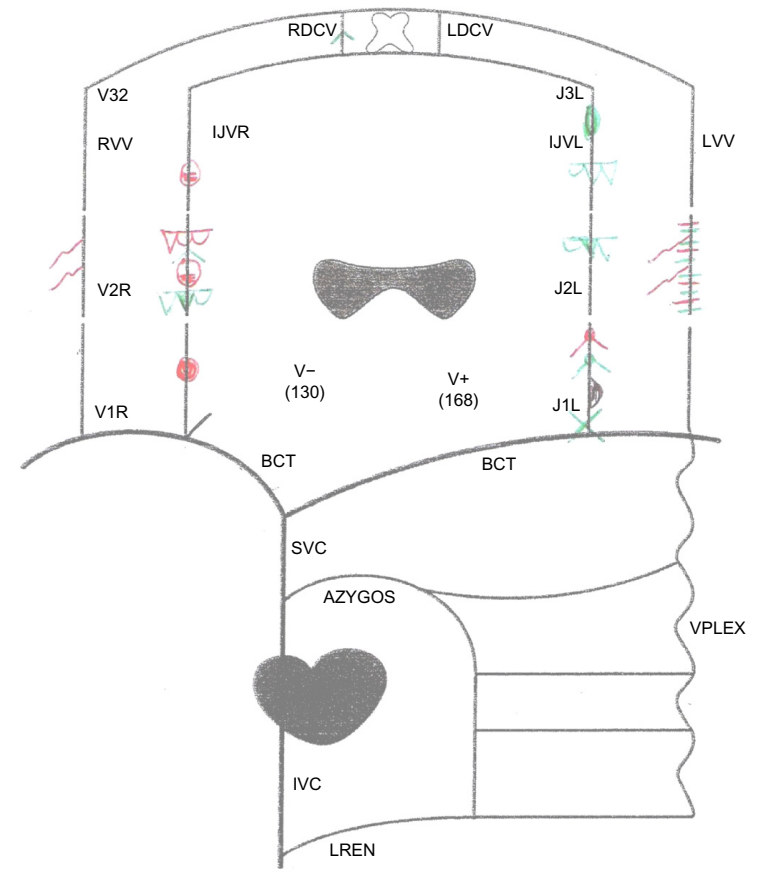

\begin{tabular}{|c|c|c|}
\hline $\begin{array}{l}\text { Zamboni criteria } \\
\square \text { Criterion } 1 \\
\square \text { Criterion } 2 \\
\square \text { Criterion } 3 \\
\square \text { Criterion } 4 \\
\square \text { Criterion } 5\end{array}$ & & $\begin{array}{l}\square \text { Type } 2 \\
\square \text { Type } 3\end{array}$ \\
\hline $\begin{array}{l}\text { ID patient: } 756 \quad \text { Sex: } F \\
\text { Age: } 33 \\
\text { Clinical type: SP } \\
\text { First symptom: optical neuryte left eye } \\
\text { Duration: } 13 \text { years }\end{array}$ & EDSS: 4 & $\begin{array}{l}\text { Score } \\
\text { CCSVI: } 1 \quad \text { CCSVI is positive if } \geq 2 \text { criteria } \\
\text { VHISS: } 2 \\
\text { MEM: } 15 \\
\text { Date: } 01 / 27 / 2013 \\
\text { Sonographer: Prof Sandro Mandolesi }\end{array}$ \\
\hline
\end{tabular}

Figure I Map on paper of an EchoColorDoppler report with morphological and hemodynamic symbols.

Abbreviations: CCSVI, chronic cerebrospinal venous insufficiency; CSA, cross-sectional area; SP, secondary progressive; EDSS, Expanded Disability Status Scale; VHISS, venous hemodynamic insufficiency severity score; MEM, hemodynamic morphological map; RDCV, right deep cerebral veins; LDCV, left deep cerebral veins; J2, internal jugular vein; RVV, right vertebral vein; LVV, left vertebral vein; VPLEX, vertebral plexus; BCT, common-brachiocephalic trunk anonymous; SVC, superior cava vein; IVC, inferior cava vein; AZYGOS, Azygos vein; LREN, left renal vein; $V_{-}$, valsalva negative; $V_{+}$, valsalva positive; JIR, lower right internal jugular vein; J2R, middle right internal jugular vein; J3R, upper right internal jugular vein; JIL, lower left internal jugular vein; J2L, middle left internal jugular vein; J3L, upper left internal jugular vein; VIR, lower right vertebral vein; V2R, middle right vertebral vein; V3R, upper right vertebral vein; VIL, lower left vertebral vein; V2L, middle left vertebral vein; V3L, upper left vertebral vein; Dx, right; Sn, left; IJVR, right internal jugular vein; IJVL, left internal jugular vein.

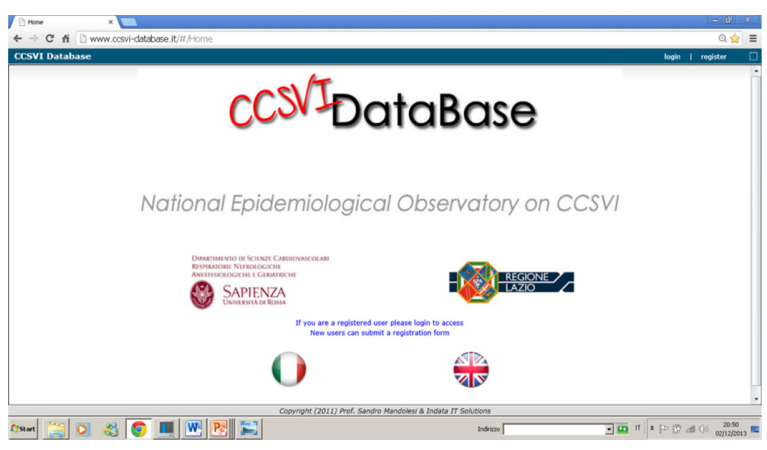

Figure 2 Web site of the National Epidemiological Observatory on CCSVI. Abbreviation: CCSVI, chronic cerebrospinal venous insufficiency. a Valsalva maneuver, a transcranial deep vein assessment, and a third ventricle measure.

The real problem in the assessment is to remember the five CCSVI criteria, which frequently causes many problems. In addition, the current ECD written reports are either oversimplified and repetitive or very complex and only comprehensible for an expert; sometimes, they are shown on complex tables that are difficult to understand. Last but not least is the current presence in the literature of two groups of criteria for ECD diagnosis of CCSVI. ${ }^{1,3}$ The first list of five criteria is the original one proposed 
Table I Symbols and terminology to be used in reporting on EchoColorDoppler map examination of the veins draining the brain

\begin{tabular}{|c|c|c|c|}
\hline & Symbol & Description & MEM score \\
\hline & Calcification & Endo- or extravascular & 0 \\
\hline & Full block & $\begin{array}{l}\text { When the block involves one or more segments with a caliber } \\
\text { equal to or greater than the previous level or the following level }\end{array}$ & 2 \\
\hline & Empty block & $\begin{array}{l}\text { When the block involves one or more segments with a caliber } \\
\text { much less than the previous level or the following level }\end{array}$ & 2 \\
\hline & Emi full block & $\begin{array}{l}\text { When the time of the drainage flow detected by pulsed Doppler is } \\
\text { longer than the stop flow on one or more segments with a caliber } \\
\text { equal to or greater than the previous level or the following level }\end{array}$ & 1 \\
\hline & Emi empty block & $\begin{array}{l}\text { When the time of the drainage flow detected by the pulsed } \\
\text { Doppler is longer than the stop flow on one or more segments } \\
\text { of size much smaller than the previous level or the following level }\end{array}$ & 1 \\
\hline & Morphological stenosis & Organic stenosis $<3 \mathrm{mmq}$ & 2 \\
\hline & Stenosis hemodynamics & Flow velocity $>150 \mathrm{~cm} / \mathrm{second}$ & 2 \\
\hline & Membrane & Hyperechoic area endovascular & 1 \\
\hline & Septum & Abnormal valve leaflet & I \\
\hline & Thickening & Valvular thickening & 1 \\
\hline & Twist & Twisting of the vessel & 1 \\
\hline & Vicarious & $\begin{array}{l}\text { Flow vicarious }>45 \mathrm{~cm} / \mathrm{second} \text { on vertebrals (V2) in clino } \\
\text { and }>60 \mathrm{~cm} \text { second in orto. Flow vicarious }>100 \mathrm{~cm} / \mathrm{second} \text { on the } \\
\text { internal jugulars (J2) both in clino and ortostatic position }\end{array}$ & 1 \\
\hline & Ectasia & Vessel diameter more than $20 \mathrm{~mm}$ & I \\
\hline & Confluence & Thickening of the confluence of jugular to subclavian & I \\
\hline & Thickening-dysplasia & Thickening (dysplasia) of the vessel wall & I \\
\hline & Thrombosis & Thrombosed segment & 2 \\
\hline \& & Recanalization & Recanalization with parietal residues & I \\
\hline & White compression & $\begin{array}{l}\text { The compression is defined as white when the vein } \\
\text { is completely compressed and we cannot detect any flow }\end{array}$ & $\begin{array}{l}2 \times \text { each } \\
\text { white }\end{array}$ \\
\hline & Frontal & & \\
\hline & Right lateral & & \\
\hline & Left lateral & & \\
\hline & Back protrusion & & \\
\hline & Black compression & $\begin{array}{l}\text { The compression is defined as black when the caliber of the vessel } \\
\text { is less than } 6 \mathrm{mmq} \text { and shows a flow }\end{array}$ & $\begin{array}{l}\text { I } \times \text { each } \\
\text { black }\end{array}$ \\
\hline & Frontal & & \\
\hline & Right lateral & & \\
\hline & Left lateral & & \\
\hline
\end{tabular}


Table I (Continued)

\begin{tabular}{lll}
\hline & Symbol & Description \\
\hline 00 & Antero protrusion & \\
& &
\end{tabular}

In the upright position, the symbol is green; compressions can be detected through the head in the front and/or side position right and/or left lateral position

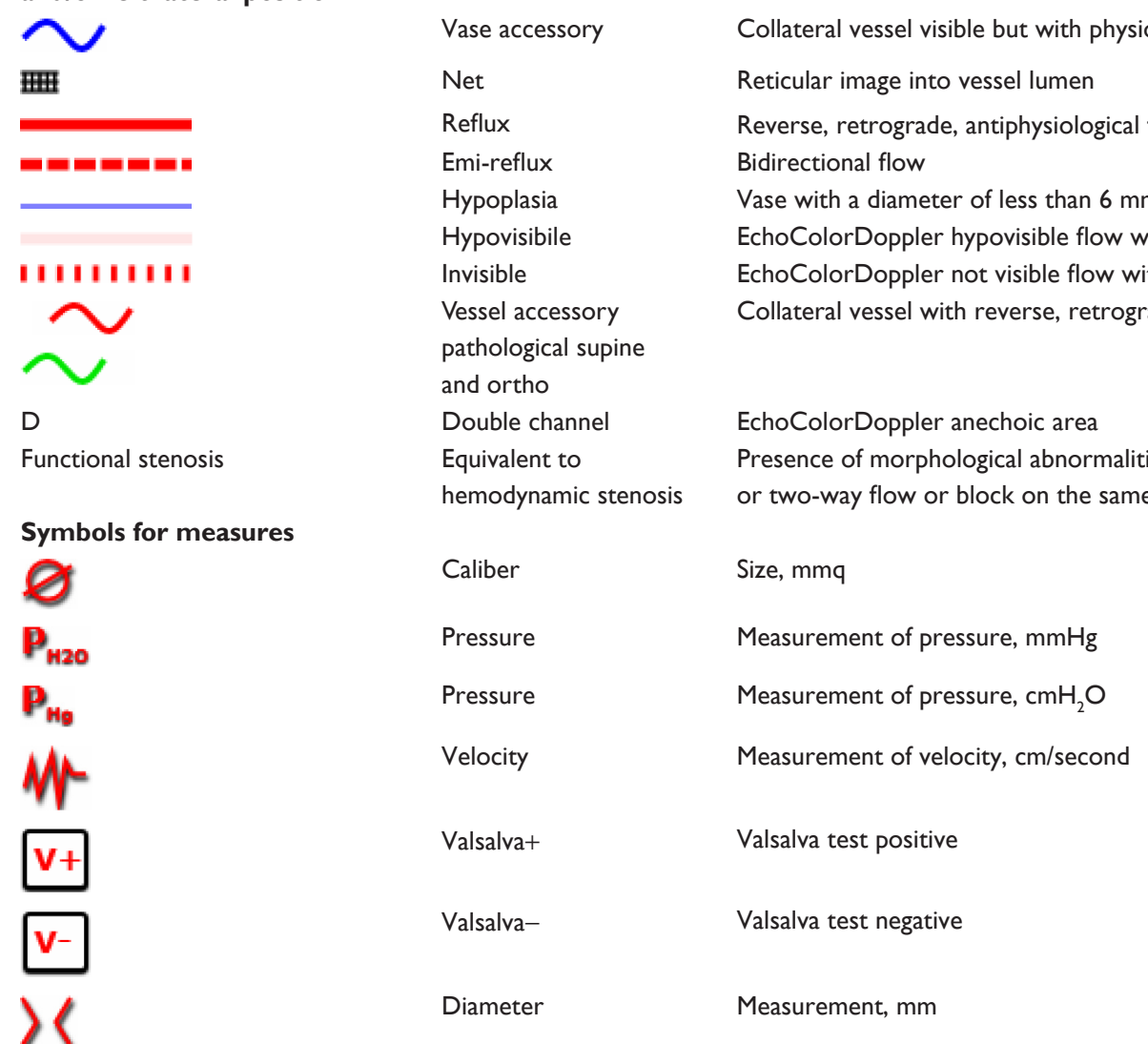

Additional symbols

$\mathbf{T}$

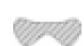

-
Free text

Thyroidectomy

Nodules

Respiratory hemodynamic index of Manconi

CSA in $\mathrm{J} 2$ inspiratory phase

CSA in subclavian inspiratory phase

Speed flow in 12 inspiratory phase

Speed flow in subclavian

inspiratory phase

Note: Consensus Conference of National Epidemiologic CCSVI Observatory, 2013. ${ }^{6}$

Abbreviations: CSA, cross-sectional area; Emi, When the time of the drainage flow detected by pulsed Doppler is longer than the stop flow; MEM, hemodynamic morphological map; PRF, pulse repetition frequency.

by Zamboni in 2009. ${ }^{3}$ The second list $^{3}$ was approved in 2011 by a consensus of experts from international scientific societies with connections to the vascular area. Sonographers find this last list of criteria very hard to keep in mind.
Since 1988, we have reported, using hemodynamic and morphologic symbols on a map, ECD examination of the veins of the lower limbs. ${ }^{2}$ We also have reported, on a map, ECD of the veins draining the brain. ${ }^{2,4}$ The first maps we used were on paper (Figure 1). Using paper to 
create the map, the possibility of mistakes in the final assessment of ECD report was lower, but human error was always possible. After 2 years of work, in 2012, we completed the Mappa Emodinamica e Morfologica (MEM-Net) program, available at http://www.mem-net. it/ (Figure 2).

MEM-net is used by the National Epidemiological Observatory on CCSVI for data collection in its computerized platform, which works on the Internet. In 2013, the observatory organized a consensus conference involving national scientific societies with connections to the vascular area to share the symbols and terminology to use for MEMnet mapping (Table 1).

The ECD map scheme is a stylized diagram of the veins draining the brain and spinal cord. (Figure 3). The internal jugular and the vertebral veins, on the map, are divided into three segments: The proximal segments are J1-V1, the medium segments are J2-V2, and the distal segments are J3-V3. J1, J2, and J3 are the segments of IJVs. J1 segments go from the confluence into the subclavian vein to the inferior level of the thyroid. J2 segments go from the inferior level of the thyroid to the jugular point; this point is where the veins cross the bifurcation of the carotid arteries. The $\mathrm{J} 3$ segment goes from the jugular point to the highest point detectable by ECD. V1, V2, and V3 are the segments of vertebral veins. The V1 segment goes from the confluence into the subclavian vein to the point in which the vertebral vein goes up from vertebral channel. The V2 segment goes from this last point to the $\mathrm{C} 3$ bone. The V3 segment is above this level.

The veins are represented by blue lines; on them are reported the morphologic and hemodynamic symbols that correspond to the assessment of ECD. The symbols of the ECD assessment done in the supine position are shown in red, and the symbols of the upright assessment are shown in green. The report on the map, by MEM-net, shortens the time of ECD assessment and reduces human error.

\section{Methods}

Using the program, when the sonographer finds, during the ECD assessment, an anomaly or a hemodynamic pathological flow, he or she immediately puts the equivalent symbol on the corresponding point of the map. A legend with all the symbols is close to the map, and you can pick which symbol you need, using the mouse (Figure 4).

To use the MEM-net program as a part of the platform of the National Epidemiological Observatory on CCSVI, you must first

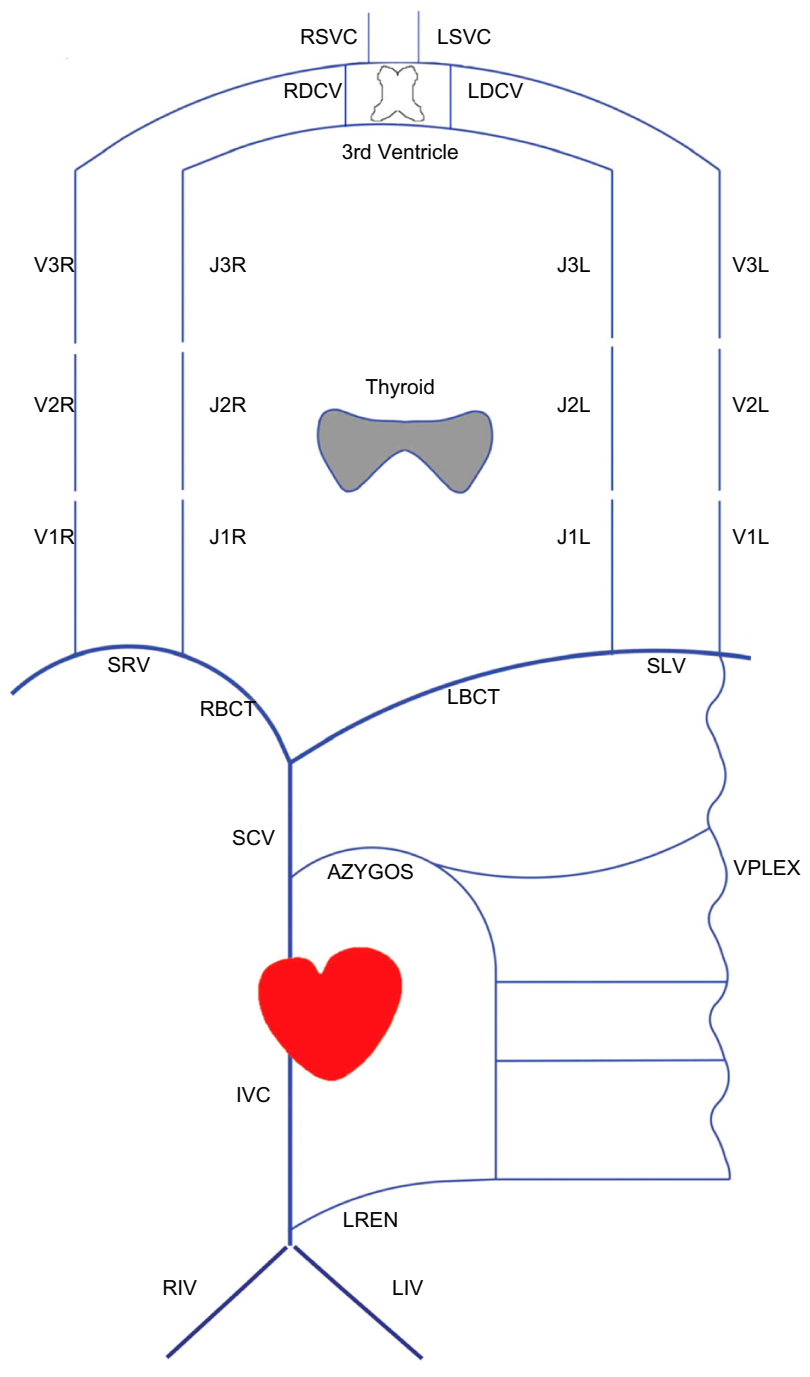

Figure 3 Map scheme for an EchoColorDoppler report.

Abbreviations: RDCV, right deep cerebral veins; LDCV, left deep cerebral veins; RVV, right vertebral vein; LVV, left vertebral vein; Vplex, vertebral plexus; RBCT, right common-brachiocephalic trunk anonymous; LBCT, left commonbrachiocephalic trunk anonymous; RSVC, right superior cava vein; LSVC, left superior cava vein; IVC, inferior cava vein; AZYGOS, Azygos vein; LREN, left renal vein; RIV, right iliac vein; LIV, left iliac vein; JIR, lower right internal jugular vein; J2R, middle right internal jugular vein; J3R, upper right internal jugular vein; JIL, lower left internal jugular vein; J2L, middle left internal jugular vein; J3L, upper left internal jugular vein; VIR, lower right vertebral vein; V2R, middle right vertebral vein; V3R, upper right vertebral vein; VIL, lower left vertebral vein; V2L, middle left vertebral vein; V3L, upper left vertebral vein; SRV, subclavian right vein; SLV, subclavian left vein; SCV, superior cava vein.

register, putting in your personal identification and password. You can then add a new patient to the archive, and the program automatically creates an ID number for privacy. Once you have entered the patient into the program, you can input the patient's personal and clinical data. Then you can enter the data on the hemodynamic page, where the symbols and the MEM-net map for the ECD report are (Figure 4). After saving the map of the ECD assessment, you can print it alone or with the written report. 


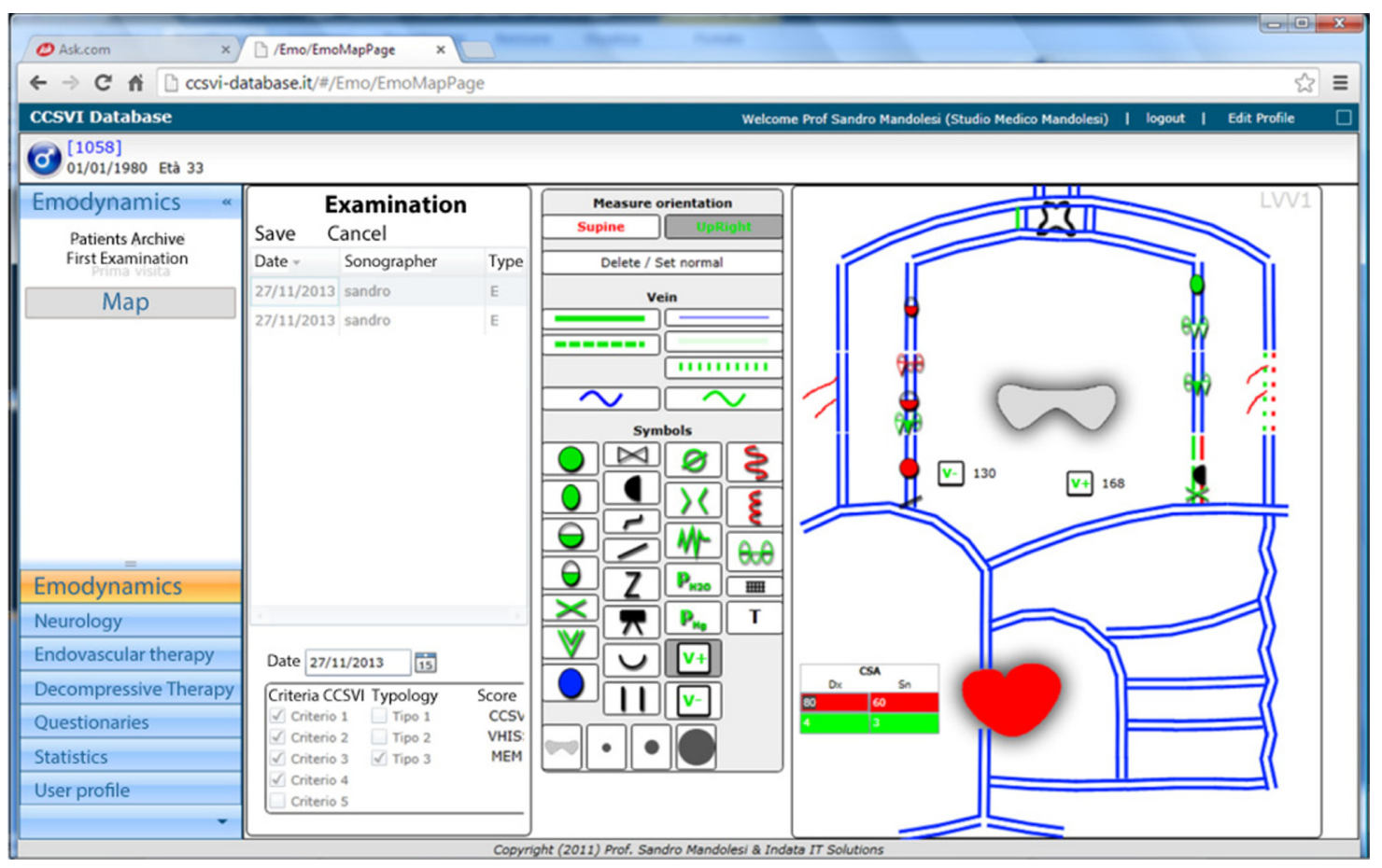

Figure 4 Page on which to digitalize the map.

Abbreviations: CCSVI, chronic cerebrospinal venous insufficiency; CSA, cross-sectional area; VHISS, venous hemodynamic insufficiency severity score; MEM, hemodynamic morphological map.

You can also use the statistic area for general or clinical data analysis of your patients (Figure 6). Creating the report on the map using MEM-net shortens the time for ECD, as reporting is done automatically (personal communication).

The MEM-net program allows all the data from a long and difficult ECD examination to be collected and, using its algorithm, made into a uniform report. The program also makes a blind control of the report, which allows its use for scientific research.

The input of the hemodynamic and morphological symbols on a MEM-net map automatically allows the following to be printed: an ECD morphological hemodynamic map with a legend of the symbols (Figure 4); examination written reporting; which criteria we founded; the CCSVI score; the venous hemodynamic insufficiency severity score; the type of CCSVI (type 1, type 2, or type 3); the clinical type (Clinically Isolated Syndrome, relapsing remitting, secondary progressive, or primary progressive); Expanded Disability Status Scale; years of disease duration; first symptom; age and sex; and an identification code for the privacy of the patient.

This software makes a faster, easier, and more appropriate ECD report of CCSVI; reduces human error; standardizes the ECD report by using shared symbols from the consensus conference; writes the assessment report (personal communication); automatically prints the report (Figure 5); saves all hemodynamic and morphological results in its database; allows 90 items of statistical analysis of patient data (Figure 6); and makes clinical and hemodynamic checks and follow-up faster and easier.

The MEM-net algorithm acts as a blind control for the ECD report and acts like an, eliminating another source of possible human error and subjective interpretation of the examination. In addition, the ECD data are collected by the same standard procedure (map report) ${ }^{5}$ the MEM-net 


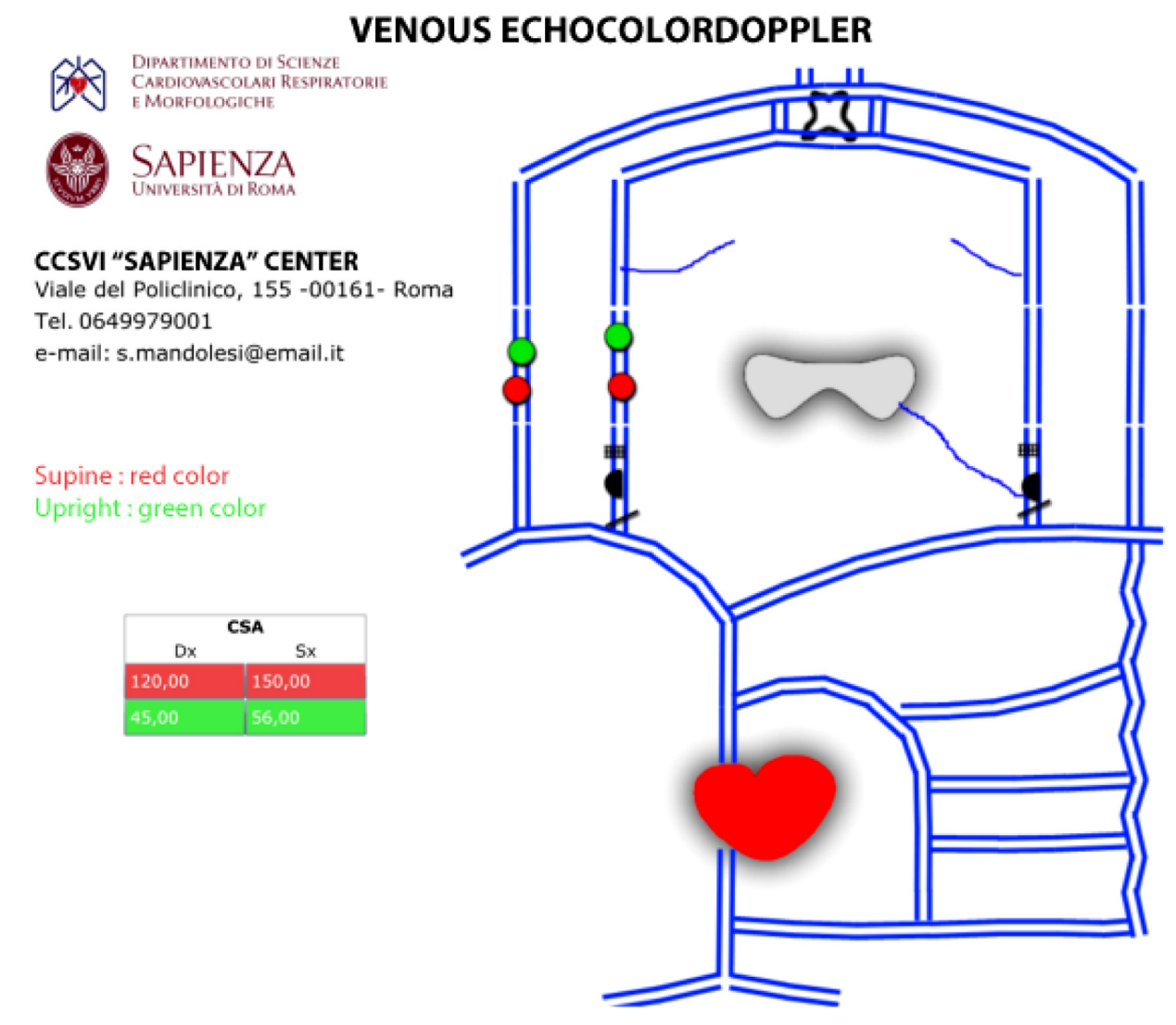

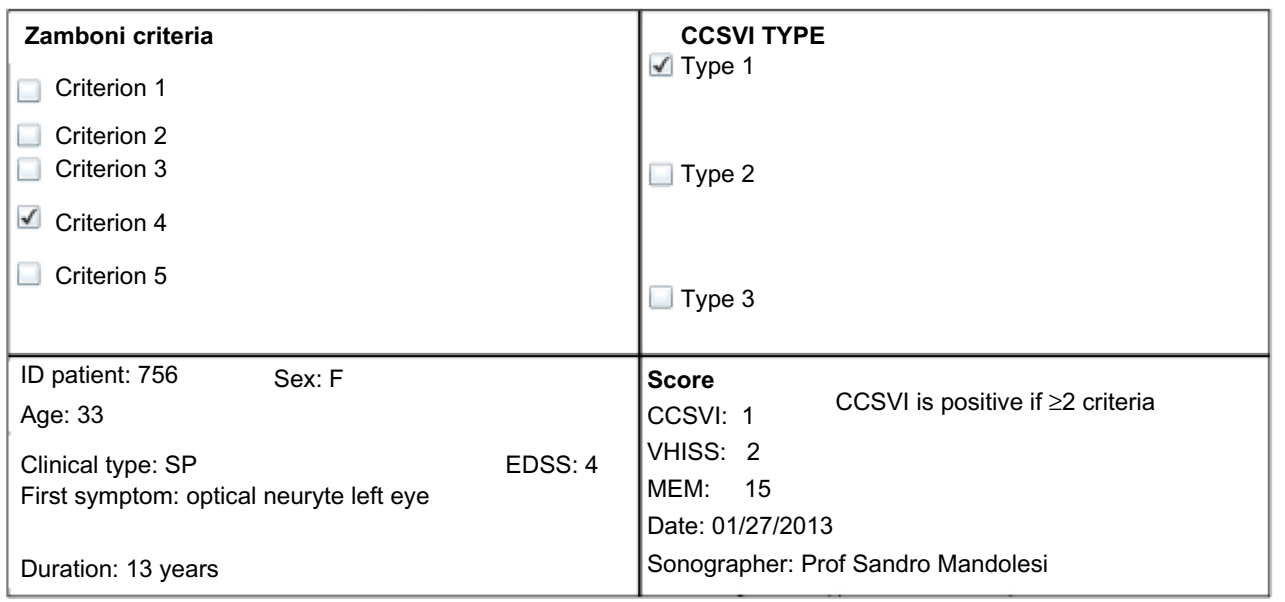

Figure 5 Report printing of an EchoColorDoppler examination with hemodynamic and morphological symbols.

Abbreviations: CCSVI, chronic cerebrospinal venous insufficiency; CSA, cross-sectional area; SP, secondary progressive; EDSS, Expanded Disability Status Scale; VHISS, venous hemodynamic insufficiency severity score; MEM, hemodynamic morphological map; Dx, left; Sx, right. 


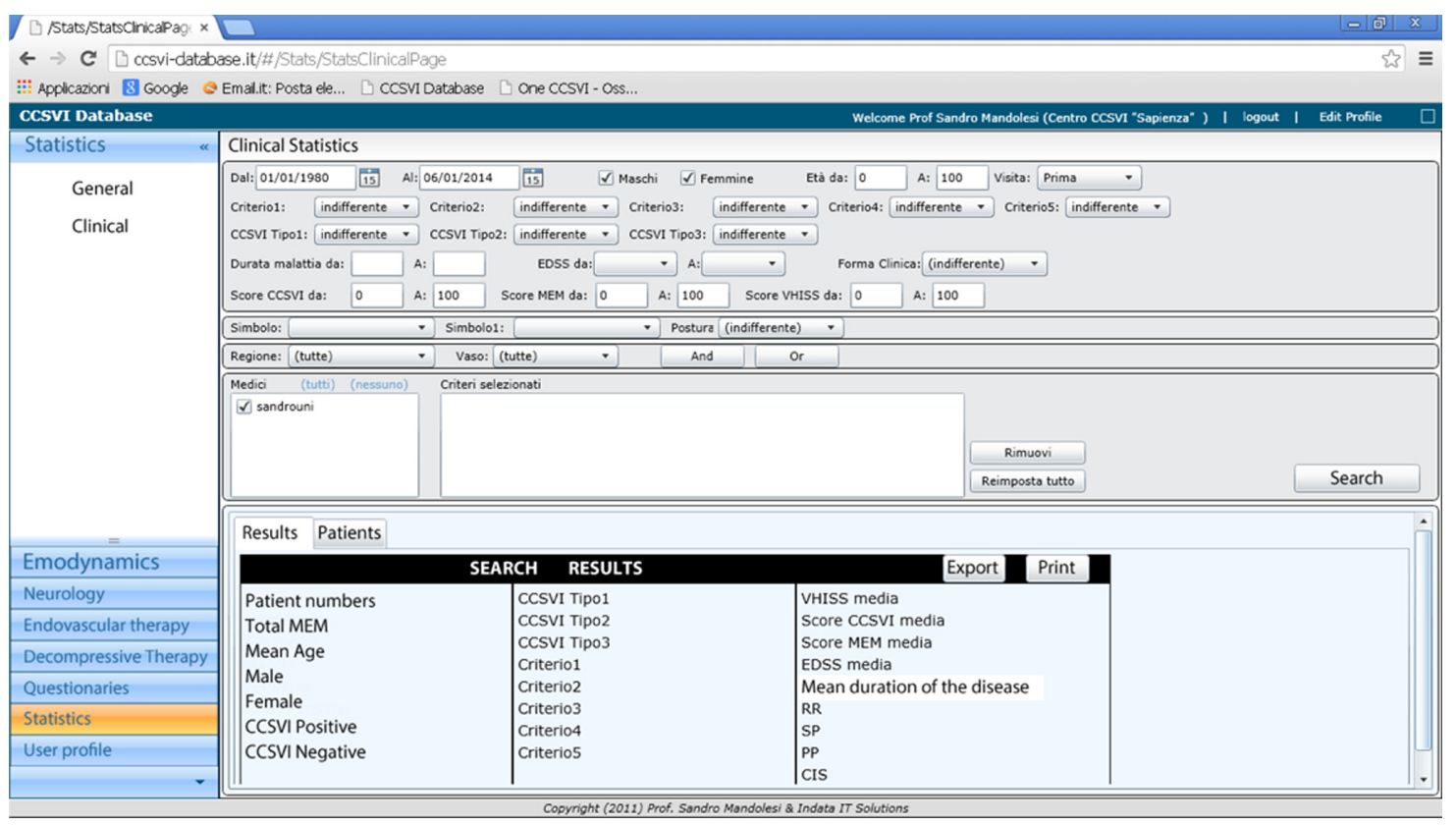

Figure 6 Page for clinical analysis.

Abbreviations: CCSVI, chronic cerebrospinal venous insufficiency; VHISS, venous hemodynamic insufficiency severity score; MEM, hemodynamic morphological map; RR, relapsing-remitting; SP, secondary progressive; PP, primary progressive; CIS, Clinically Isolated Syndrome; EDSS, Expanded Disability Status Scale.

program also allows research on homogeneous samples in short time.

\section{Conclusion}

Using the ECD map report will change the current reporting habits of sonographers because they normally write the report at the end of the examination, but the computed map MEMnet program must be used throughout the ECD assessment. To overcome this problem, the National Epidemiological Observatory on CCSVI schedules 1 day practical work classes to teach reporting on the ECD examination for CCSVI map. Participants can review the course on the observatory website (http://www.osservatorioccsvi.org/; Figure 2).

We hope that in the future, everyone will use this data collection tool for all scientific work on this topic.

\section{Disclosure}

The authors report no conflicts of interest in this work.

\section{References}

1. Zamboni P, Galeotti R, Menegatti E, et al. Chronic cerebrospinal venous insufficiency in patients with multiple sclerosis. J Neurol Neurosurg Psychiatry. 2009;80(4):392-399.

2. Francesch CI. Théorie et Practique de la Cure Conservatrice et Hemodynamique de L'insuffisance Veineuse en Ambulatoire. [Theory and practice of the conservative ambulatory cure of venous insufficiency]. France: Editions De L'armançon; 1988. French.

3. Zamboni P, Morovic S, Menegatti E, Viselner G, Nicolaides AN. Screening for chronic cerebrospinal venous insufficiency (CCSVI) using ultrasound - recommendations for a protocol. Int Angiol. 2011;30(6): 571-597.

4. Galeandro AI, Quistelli G, Scicchitano P, et al. Doppler ultrasound venous mapping of the lower limbs. Vasc Health Risk Manag. 2012;8:59-64.

5. Antignani PL, Gossetti B, Righi D, et al. Accreditation process for the performance of ultrasound vascular diagnostics procedures. Minerva Cardioangiol. 2005;53(5):485-506.

6. Mandolesi S, D'Alessandro A, Manconi E, et al. Symbolism and terminology of EchoColorDoppler map reporting of the main veins draining the brain - recommendations for a protocol. Acta Phlebologica. 2014 In press.
Journal of Vascular Diagnostics

\section{Publish your work in this journal}

Journal of Vascular Diagnostics is an international, peer-reviewed journal of diagnostics, focusing on non invasive vascular investigation methods involved in the evaluation of vascular diseases. The journal is committed to the rapid publication in the fields of vascular diseases. Original research, review, case reports, expert opinion and commentaries

\section{Dovepress}

are all considered for publication. The manuscript management system is completely online and includes a very quick and fair peer-review system, which is all easy to use. Visit http://www.dovepress.com/testimonials.php to read real quotes from published authors. 